\title{
Clinical Course of Atopic Dermatitis in an Adult with Amyotrophic Lateral Sclerosis: Aetiological Implications of Voluntary Movements and Dermatitis Severity
}

Tatsushi KIYOHARA ${ }^{1,2}$, Takayasu FUKUDOME ${ }^{3}$, Yoshiyuki KAMIO ${ }^{2}$, Yuta KOIKE² and Hiroyuki MUROTA ${ }^{2}$

${ }^{1}$ Department of Dermatology and ${ }^{3}$ Department of Neurology, National Hospital Organization Nagasaki Kawatana Medical Center, Kawatana, and ${ }^{2}$ Department of Dermatology, Nagasaki University Graduate School of Biomedical Sciences, 1-7-1, Sakamoto, Nagasaki City, 852-8501 Nagasaki, Japan. E-mail: h-murota@nagasaki-u.ac.jp

Accepted Dec 15, 2021; Epub ahead of print Jan 28, 2022

Acta Derm Venereol 2022; 102: adv00644. DOI: 10.2340/actadv.v102.1121

Atopic dermatitis (AD) is characterized by symmetrical, pruritic irritation of the skin. Although the pathogenesis of $\mathrm{AD}$ involves skin barrier dysfunction, inflammation, and an itch-scratch cycle $(1,2)$, the contribution of each remains obscure. We report here an adult case of $\mathrm{AD}$ accompanied by amyotrophic lateral sclerosis (ALS) in which the $\mathrm{AD}$ manifestations were limited to the areas of voluntary movement.

\section{CASE REPORT}

A 52-year-old woman was referred to our dermatology clinic for AD. She had a history of uterine myoma and no family history of $\mathrm{AD}$. She was diagnosed with $\mathrm{AD}$ at 20 years of age, for which she received only topical glucocorticoids. She developed ALS 1 year before the first visit to our clinic.

On initial dermatological examination, the patient exhibited cheilitis, slight facial erythema, and skin dryness (Fig. 1A). The erythema was apparent on her eyelids. There were no other dermatological findings. Notably, she objectively rated her pruritus as "no itch." Her Scoring Atopic Dermatitis score was 15.1, and her Eczema Area and Severity Index score was 0.7. Results of the patient's blood tests are summarized in Table I. Based on her mild disease, we prescribed topical moisturizers and mild topical glucocorticoids, instead of a more potent therapy (e.g. potent topical glucocorticoids, immunosuppressives).

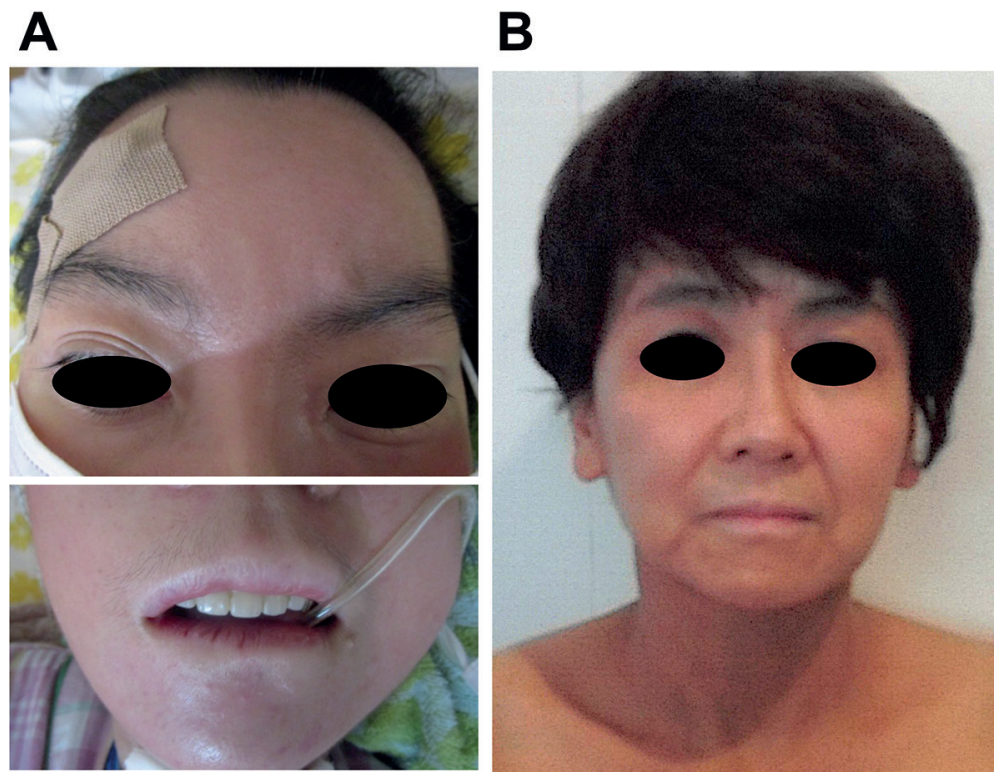

Fig. 1. Patient photographs. (A) Photograph of the patient at 52 years of age, obtained during the first consultation at our dermatology clinic. (B) Photograph of the patient at 47 years of age, taken by the patient's family.
Table I. Laboratory results during the first hospital visit

\begin{tabular}{|c|c|c|}
\hline Items & Measurement & Reference \\
\hline Number of eosinophils $(/ \mu \mathrm{l})$ & 201 & NA \\
\hline Total IgE (IU/ml) & 5,050 & $<170$ \\
\hline \multicolumn{3}{|l|}{ Antigen-specific IgE (class) } \\
\hline Cedar pollen & 6 & 0 \\
\hline Cupressaceae & 2 & 0 \\
\hline Alternaria & 2 & 0 \\
\hline Shrimp & 2 & 0 \\
\hline Mugwort & 2 & 0 \\
\hline $\operatorname{TARC}(\mathrm{pg} / \mathrm{ml})$ & 394 & $<450$ \\
\hline C-reactive protein $(\mathrm{mg} / \mathrm{dl})$ & 0.6 & $<0.2$ \\
\hline
\end{tabular}

TARC: Thymus and activation-regulated chemokine; NA: not applicable.

Regarding the patient's dermatological history, we confirmed the clinical course of her AD by both gathering clinical information from her former attending physician and reviewing photographs taken by her family. In her medical records at the age of 47 years ( 5 years before presenting to our clinic), she had experienced persistent symptoms of moderate to severe AD since the age of 20 years. Because her attending physician was not a dermatologist, her medical records did not include descriptions of her specific skin phenotype. Therefore, we considered her skin symptoms at that time based on a photograph provided by her family: Erythema with lichenification was diffusely distributed on her head and neck (Fig. 1B), and lichenoid dermatitis with seropapules was observed on her arm in another photograph (not shown, due to data protection). Based on her treatment history, the AD symptoms went untreated. On reviewing the patient's neurological history, we noted that her ALS presented at the age of 51 years with dysphagia. Initially, sweets tended to become stuck in her throat; later, she would choke while drinking water. After onset, her ALS advanced quickly. At the time she was initially evaluated in our dermatology clinic, she was hospitalized for respiratory management. Her voluntary locomotion was limited to her fingers and face, and her extraocular muscle function was reduced.

The patient's extent and severity of muscle dysfunction had dramatically increased by the time of presentation at our clinic, and her AD manifestations had significantly decreased despite normal skin sensations. Specifically, her AD improved in areas of impaired voluntary muscle function. The previously chronic, intractable dermatitis on her arms had disappeared with the development of hypofunction of the respiratory muscles, triceps, and biceps brachii muscles.

\section{DISCUSSION}

ALS is a relatively commonmotor neurone disease caused by degeneration of the up- 
per and lower motor neurone corticospinal tracts. ALS is characterized by symptoms related to bulbar palsy and skeletal muscle weakness, while tactile, nociceptive, and itch sensations, as well as bladder and rectal functions, remain normal. Inflammatory responses are enhanced in patients with ALS due to motor neurone cell death. Innate and acquired immune cells in the perineuronal area, and monocytes and natural killer cells in circulating blood are activated. In addition, the loss of regulatory $\mathrm{T}$ cell functions results in persistent inflammation (3). Furthermore, increased plasma concentrations of eotaxin-1, which has eosinophilotactic activity, are found in patients with ALS (4). Thus, immune responses seen in these patients probably lead to exacerbation of $\mathrm{AD}$, but, in our case, there was no exacerbation of the dermatitis.

Regarding skin disorders related to ALS, except for 1 study describing dyshidrotic eczema in an affected area (5), there do not appear to be any reports of AD accompanied by ALS. Thus, we speculate that the elimination of scratching behaviour caused by ALS, decreased voluntary muscle function, or extension and flexure of the skin may have contributed to decreases in itch intensity and disease severity observed in our patient. Several reports support this premise. Troilius et al. (6) reported 5 patients with hemiplegia who only developed eczema in non-paralysed areas. Furthermore, Azimi et al. (7) focused on the underlying relationship between dermatoses and neurological deficits, and performed a literature review for cases with fluctuations in dermatoses after the development of neurological disorders. Their review found that of 23 cases of dermatoses (e.g. eczema, psoriasis, scleroderma, rosacea, contact dermatitis, and bullous pemphigoid), 19 experienced the complete disappearance of skin symptoms in areas served by the damaged nerve. Moreover, misfolded protein aggregation in the skin can accompany certain neurodegenerative disorders, such as ALS, suggesting the relevance of a "brain-skin connection" (8). Although a pathological analysis was not performed in the current case, the disappearance of AD in ALS-affected areas appeared to be strongly associated with motor neurone, but not sensory neurone, dysfunction. Although this hypothesis seems to contradict the disappearance of itch in the current case, it could be argued that voluntary motion is the trigger for itching in cases of AD.

Pruritic diathesis in cases of AD impedes long-term disease control. Itching may occur with both touch and nociceptive stimuli (e.g. temperature, sweat, clothes) in $\operatorname{AD}(9,10)$ and is difficult to treat. In our patient, the chronic dermatitis regressed spontaneously in areas of impaired voluntary motion. This observation suggests that skin movement may contribute to disease persistence in $\mathrm{AD}$, either directly or by triggering pruritus as a form of alloknesis (11). Admittedly, this hypothesis is based on a single patient, for whom we were unable to obtain detailed records of her AD disease course. However, the current report provides useful information to shape our thinking about the pathogenesis of $\mathrm{AD}$, especially the reasons for its typical distribution and the presence of alloknesis in patients with this disorder.

\section{REFFERENCES}

1. Katoh N, Ohya Y, Ikeda M, Ebihara T, Katayama I, Saeki H, et al. Clinical practice guidelines for the management of atopic dermatitis 2018. J Dermatol 2019; 46: 1053-1101.

2. Mack MR, Kim BS. The itch-scratch cycle: a neuroimmune perspective. Trends Immunol 2018; 39: 980-991.

3. Appel SH, Beers DR, Zhao W. Amyotrophic lateral sclerosis is a systemic disease: peripheral contributions to inflammationmediated neurodegeneration. Curr Opin Neurol 2021; 34 : 765-772.

4. Huber AK, Giles DA, Segal BM, Irani DN. An emerging role for eotaxins in neurodegenerative disease. Clin Immunol 2018; 189: 29-33.

5. Nakai K, Yoneda K, Moriue T, Hosokawa Y, Yokoi I, Kubota Y. Unilateral palm pompholyx in a patient with amyotrophic lateral sclerosis. Eur J Dermatol 2011; 21: 445-446.

6. Troilius A, Möller H. Unilateral eruption of endogenous eczema after hemiparesis. Acta Derm Venereol 1989; 69: 256-258.

7. Azimi E, Lerner EA, Elmariah SB. Altered manifestations of skin disease at sites affected by neurological deficit. $\mathrm{Br} J$ Dermatol 2015; 172: 988-993.

8. Clos AL, Kayed R, Lasagna-Reeves CA. Association of skin with the pathogenesis and treatment of neurodegenerative amyloidosis. Front Neurol 2012; 3: 5.

9. Murota $\mathrm{H}$, Katayama I. Exacerbating factors of itch in atopic dermatitis. Allergol Int 2017; 66: 8-13.

10. Thyssen JP, Rinnov MR, Vestergaard C. Disease mechanisms in atopic dermatitis: a review of aetiological factors. Acta Derm Venereol 2020; 100: adv00162.

11. Andersen $\mathrm{HH}$, Akiyama T, Nattkemper LA, van Laarhoven $A$, Elberling J, Yosipovitch G, Arendt-Nielsen L. Alloknesis and hyperknesis-mechanisms, assessment methodology, and clinical implications of itch sensitization. Pain 2018; 159: 1185-1197. 